\title{
How much color do we see in the blink of an eye? \\ Michael A. Cohen ${ }^{1,2} \&$ Jordan Rubenstein ${ }^{2}$ \\ ${ }^{1}$ McGovern Institute for Brain Research, Department of Brain and Cognitive Sciences, Massachusetts Institute of Technology \\ ${ }^{2}$ Department of Psychology and Program in Neuroscience, Amherst College
}




\begin{abstract}
Visual experience is painted in color. A change in hue or saturation can dramatically alter our understanding of a scene and how we feel about it. Subjectively, color does not feel like an optional dimension to be extracted only when necessary, but an automatically represented property of our entire visual field. Here, we ask whether that subjective impression is true. Using a variant of an inattentional blindness paradigm, we showed observers snapshots of colorful scenes when unbeknownst to them, an image was presented that was either desaturated or hue rotated across an overwhelming majority of the images. Although observers fixated on these images long enough to identify and describe them, a large number of observers were completely unaware of these drastic color manipulations. These findings suggest that the amount of color observers perceive "in the blink of an eye" is drastically less than personal introspection would suggest.
\end{abstract}


How colorful is visual experience? At first glance, this question seems so simple that it is natural to assume the answer is already known. Surprisingly, this is not the case. While the functional organization of color processing has been thoroughly characterized in both the retina and the brain (Conway, 2009), how this organization relates to perception is the subject of considerable debate. On one hand, intuition suggests that observers have a rich colorful experience that extends well into the periphery. On the other hand, many researchers argue that this intuition is mistaken and cite paradigms like change blindness (Rensink et al., 1997) and inattentional blindness (Mack \& Rock, 1998) to argue that observers overestimate the amount of color they perceive (Dennett, 1991; Lau and Rosenthal, 2011). Conversely, other researchers challenge these claims by arguing that subjective introspection is correct and observers do enjoy a rich colorful experience (Block, 2007; Haun et al., 2017; Tyler, 2015).

Here, we sought to make progress on this issue by focusing on characterizing how much color observers perceive in a single "snapshot." We showed observers a series of large colorful scenes (i.e., $26^{\circ}$ of visual angle) and asked them to make simple judgments about those scenes (i.e., Did it have a face? Was it an indoor or outdoor scene?). Then, unbeknownst to them, we presented a target image that had either been 1) entirely desaturated or 2) hue rotated $180^{\circ}$ in the periphery. In both cases, the only portion of the scenes that were not altered was the central foveal/parafoveal part of the image, which subtends a radius of $4^{\circ}$ of visual angle (Polyak, 1941; Swanson \& Fish, 1995; Millodot, 2014). To put that number in perspective, the fovea/parafovea comprises less than $1 \%$ of the visual field in everyday life.

How often will observers notice these altered stimuli? Surprisingly, even though observers fixated on these images for almost $300 \mathrm{~ms}$, a substantial percentage of them were oblivious to the fact that they looked directly at desaturated or hue rotated images. However, it should be stressed that observers did perceive many other aspects of those target images. Indeed, the vast majority of participants could describe the target image and pick it out of a lineup, they were simply unaware that the periphery of the target image had been radically altered. In addition, the size of this effect varied as a function of the primary task observers were performing. When observers had their attention focused on looking for a face, close to three quarters of participants did not notice the altered stimuli. Moreover, even when observers were making an indoor/outdoor judgment about the scene images, and attention was more distributed, close to a third of observers still did not notice the altered stimuli. Together, these results suggest that a "snapshot" of perceptual experience contains surprisingly little color.

\section{Methods}

\section{Participants}

160 participants were run across four experimental conditions (40 different participants per condition). Each participant was assigned to one specific experimental condition: 1) face task with a desaturated critical stimulus, 2) scene task with a desaturated critical stimulus, 3) face task with a hue rotated critical stimulus, and 4) scene task with a hue rotated critical stimulus. All participants were recruited from the Amherst College and 
Massachusetts Institute of Technology communities. All participants gave informed consent and were compensated with $\$ 5$ or course credit. All experimental procedures were approved by the Committee on the Use of Human Subjects in Research under the institutional review board of Amherst College.

\section{Equipment}

All were presented on a 27 -inch $\mathrm{LCD}$ monitor with a $60 \mathrm{~Hz}$ refresh rate. A chin and forehead rest was used to minimize head movements and maintain a constant viewing distance of $57 \mathrm{~cm}$. All experiments were created using MATLAB and the Psychophysics toolbox (Brainard, 1997; Pelli, 1997). The background color of the screen was gray with a luminance of $68.5 \mathrm{~cd} / \mathrm{m}^{2}$. For the scene tasks, a gazepoint GP3 Eye Tracker was used to monitor the position of both eyes in real time. All responses on the first 10 trials and the 4 alternative forced choice task were made with button presses on a keyboard. Verbal responses were recorded by the experimenter for the critical trials.

\section{Stimuli}

All stimuli subtended $26^{\circ}$ of visual angle and were acquired through online databases (i.e., SUN database; Xiao et al., 2010), Google Image Search, and personal photograph collections of the authors.

Face task stimuli: For the face detection task, there were 50 face target images and 50 distractor images (Figure 1a). Each target image had a human face that was unambiguously in the middle of the image viewed either head on or from the side. Distractor image were defined by not having a human face in the middle, although they could have animal faces in the middle or humans in the periphery. All images comprised a wide variety of settings and locations (e.g., a woman swimming, a farmer working, two dogs sitting together, etc.).

a) Example stimuli from face task

Face targets
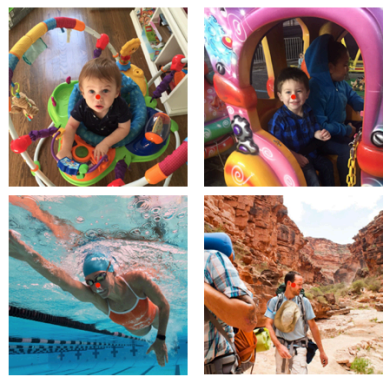

No face distractors

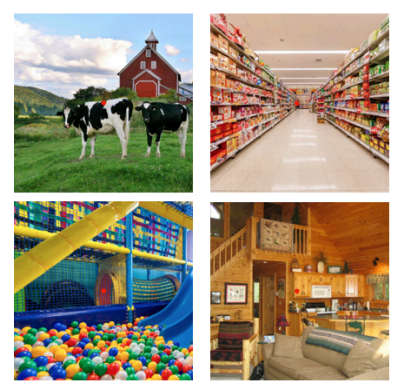

b)

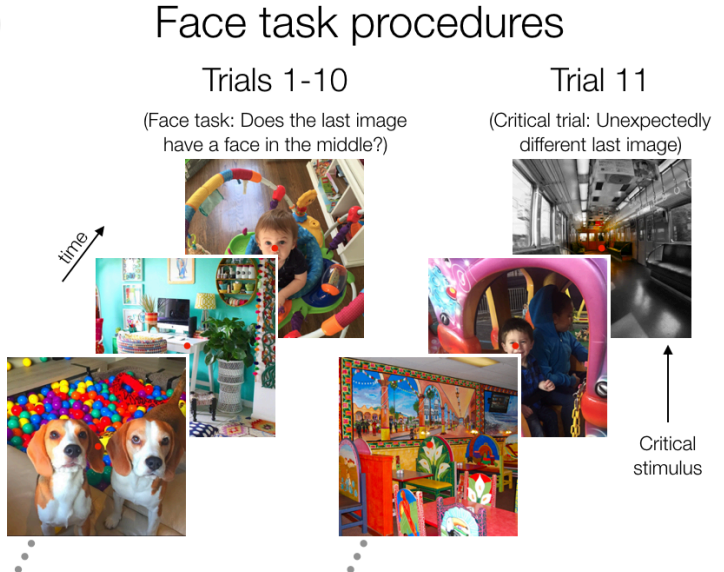

Figure 1. a) Representative examples of the different types of stimuli used on the face task. b) Representation of the trial sequence for the face task procedures. The first 10 trials were for the face task and the critical trial occurred on the $11^{\text {th }}$ trial. On this trial, the last image in the stream was unexpectedly replaced by a desaturated or hue rotated image.

Scene task stimuli: For the indoor/outdoor task, the same stimuli were used as in the face task and the procedures used to select the stimuli from trial to trial were identical as 
the face task (Figure 2a). Thus, the stimuli did not differ between the tasks; only the tasks being performed on those stimuli were different. The lone exception to this rule came in selecting the stimuli that observers made judgments about. In some cases, we decided that certain images were ambiguous in terms of whether or not they were indoors or outdoors (e.g., a woman in a green house). To avoid confusion, observers were never asked to make judgments about those particular images. However, on the critical trial, in which an altered target image was presented, no stimuli were excluded from consideration. The same stimulus batch and the same selection procedures used in the face task were used in the scene task.

a) Example stimuli from scene task Indoor scenes
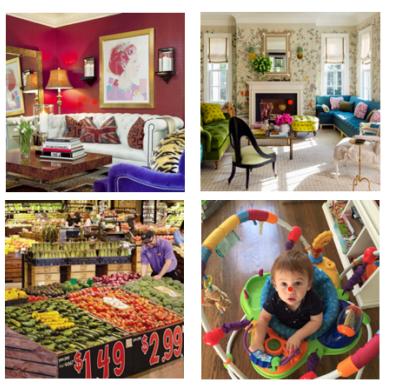

Outdoor scenes

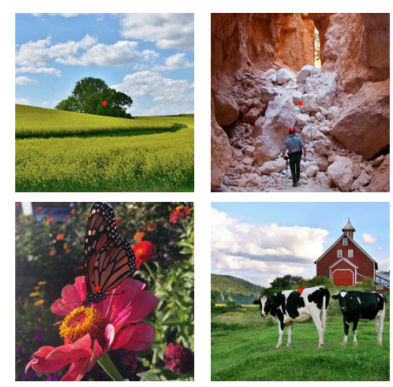

b)

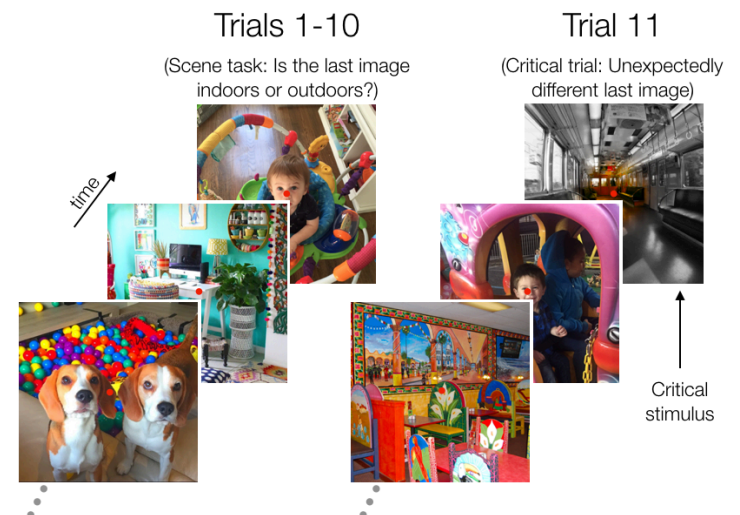

Figure 2. a) Representative examples of the different types of stimuli used on the scene task. d) Representation of the trial sequence for the scene task procedures. The first 10 trials were for the scene task and the critical trial occurred on the $11^{\text {th }}$ trial. On this trial, the last image in the stream was unexpectedly replaced by a desaturated or hue rotated image.

Critical stimuli: For all critical stimuli, the center of the image was preserved by a flat top Gaussian that subtended $8^{\circ}$ of visual angle (diameter). The flat top portion of the Gaussian subtended $5^{\circ}$ of visual angle, which approximately corresponds to the fovea, and was entirely unaltered. Meanwhile, the slopes of the Gaussian served as a gradual fade from the untouched center of the image to the altered periphery of the image (i.e., desaturated or hue rotated). This sloping/faded region comprised the remaining portion of the preserved region and landed approximately on the parafovea of the retina. For the desaturated stimuli, the foveal/parafoveal part of the image was colored, while the rest of the region was entirely desaturated (i.e., black and white). For the hue rotated critical stimuli, all images were put into CIELAB color space and rotated $180^{\circ}$ (see Figure 3 and Supplementary Materials for several example critical stimuli and the procedures used to generate those stimuli). It should be noted that only a subset of stimuli were selected to be included as critical stimuli in the hue rotated condition. In many cases, rotating the images $180^{\circ}$ resulted in images for which it was not immediately obvious that any rotation had occurred (e.g., a woman standing in front a modern art painting, a child in a ball pit). For many images, the colors of the items in the periphery were primarily on objects for which the color of those items was somewhat arbitrary (e.g., a piece of modern art can be any color, balls in a ball pit can be any color). Thus, only stimuli for which the items in the periphery were a natural, diagnostic color (e.g., the wood of a construction site, the grass of a baseball field, or the water in a clean pool) were used in the hue rotation condition as critical stimuli. ${ }^{1}$ 
Original
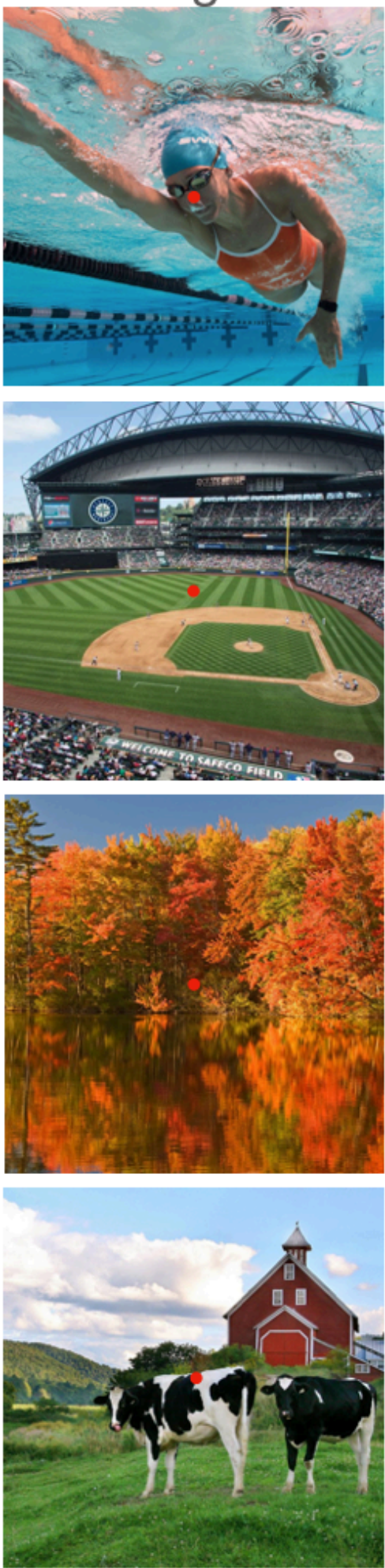

Desaturated
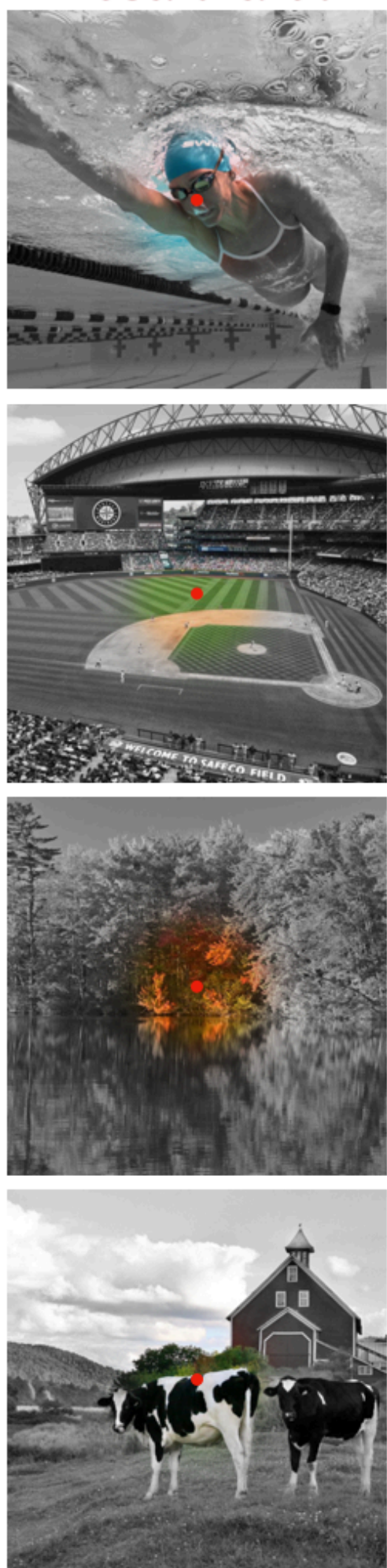

Hue rotated
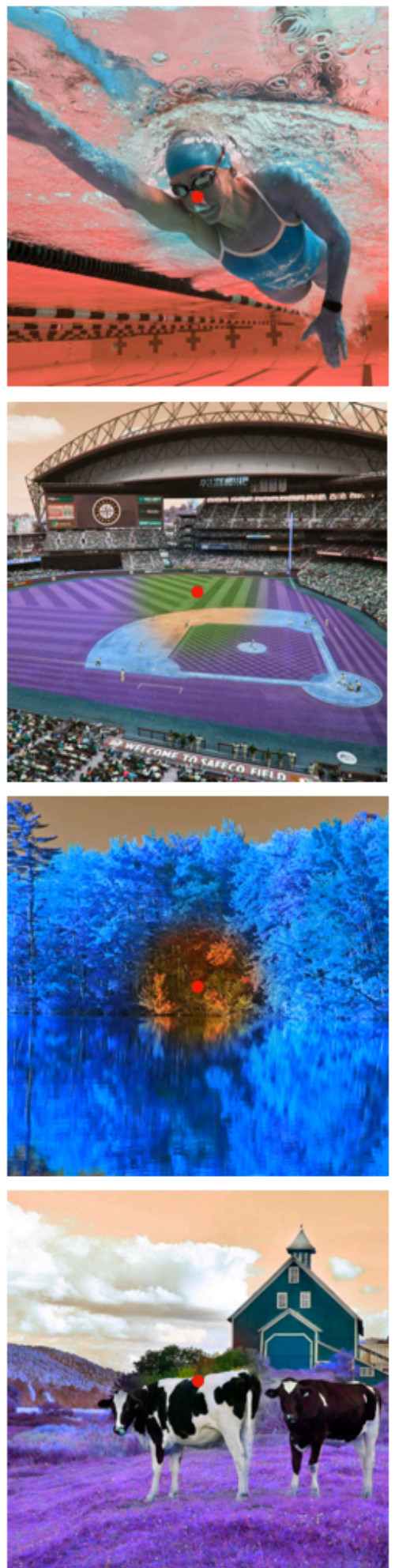

Figure 3. Examples of an original stimulus (left column), a desaturated critical stimulus (center column), and a hue rotated critical stimulus (right column). See Supplementary Materials for several more examples. 


\section{Procedures}

For every experiment, the first 10 trials were on the primary task (i.e., the face or scene task). On every trial, observers were shown 7-30 images. Each image was shown for 288 $\mathrm{ms} / \mathrm{item}$ with a $100 \mathrm{~ms}$ gap in between. We chose a presentation rate of $288 \mathrm{~ms} / \mathrm{item}$ because this approximates the duration of a single fixation period in naturalistic viewing conditions (Hayhoe et al., 2003; Henderson, 2003; Nuthmann, 2017; Pelz et al., 2001; Rayner, 1998; Rayner et al., 2011; Unema et al., 2005). For every experiment, regardless of whether or not the trial had a face at the end of it, there were between 2-5 images that had faces in the middle throughout the stream. With the face task, participants were told that this would happen and that they only had to say whether or not the last image had a human face in the middle. All images were randomly selected from trial to trial from the different stimulus folders (i.e., "face targets," "no face distractors," etc.). No image was ever shown twice within a trial, although images could be shown multiple times across trials. For the first 10 trials of the face task, half of the trials had a face target present at the end and half did not. For the first 10 trials of the scene task, half of the trials had an indoor scene at the end and half had an outdoor scene at the end. At the end of each trial, a screen appeared that prompted the participant to report whether or not the last image had a human face in the middle or was an indoor/outdoor scene.

The critical trial always occurred on trial 11 . On these trials, the selection criteria for the stimuli presented before the critical stimulus was identical to all previous trials. The main difference was that the very last image in the stream was a critical target stimulus. For the face task, half of the participants saw a critical stimulus with a human face in the middle and the other half saw a critical stimulus that did not have a human face in the middle. For the scene task, half of the participants saw a critical stimulus of an indoor scene and the other half saw a critical stimulus of an outdoor scene. In all conditions, as soon as the critical stimulus disappeared, the display went blank and the experimenter immediately began asking questions to the participant. Those questions were:

1) "Did you notice anything strange or different about that last trial?"

2) "If I were to tell you there was something different about that last trial, could you tell me what it was?"

3) "If I were to tell you there was something different about the very last image you saw on that last trial, could you tell me what it was?"

If participants could not correctly identify the critical alteration after those questions, participants were told what had occurred and then performed a four alternative forced choice task where they were asked to identify the specific image they saw. Showing four alternatives like this served two purposes: First, it allowed us to show participants the modifications we made to the images and verify that they did not notice those modifications. Second, by showing four examples of critical stimuli, we could also ask observers to identify which image they had seen. Having participants identify the last image they saw is important because if participants could not even accurately recognize the last image they were shown, their inability to notice alterations to the stimuli would be difficult to interpret. For those observers that were able to describe the critical alteration at 
any point during the questioning, the questioning stopped and participants immediately performed the four alternative forced choice task.

For the four alternative forced choice task, observers saw four stimuli that had been altered in the same way (i.e., four desaturated or hue rotated images). For the face task, all four images contained a face in the middle or none of them did depending on the specific stimulus participants saw on the last trial. For the scene task, all four images would either be of an indoor or outdoor scene depending on the specific stimulus participants saw on the last trial. Upon being shown those four images, participants were told what had happened on the last trial and were asked to confirm whether or not they had noticed. Only those participants who claimed to not notice the critical manipulation when seeing the four images were classified as being inattentionally blind. Every participant, regardless of their inattentional blindness score, was asked to select the last image they believe they saw on the last trial.

One important difference between the face and scene tasks was the use of an eyetracker in the scene task, which was used to ensure participants maintained fixation throughout the trial. Given the task demands of the face task, it seemed safe to assume participants maintained fixation throughout. In fact, if participants did not, that would only decrease our inattentional blindness rates since participants are more likely to notice the critical target stimuli if they foveate desaturated or hue rotated parts of the image. For the scene task, participants performed a brief calibration sequence at the beginning of the experiment and would recalibrate if there were problems with the eyetracking system. Once a trial began, if participants broke fixation at any point, the trial would break and then start over after participants reestablished fixation.

\section{Results}

The results from all experimental conditions are plotted in Figure 4. With desaturated critical stimuli, $70 \%$ of participants (28/40) were unaware of the desaturated stimuli during the face task. Meanwhile, $33 \%$ of participants (13/40) were unaware of the desaturated stimuli during the scene task. In this case, there was a significant difference in inattentional blindness rates depending on the task $\left(X^{2}(1)=9.81, P<0.01\right)$. 
Inattentional blindness data

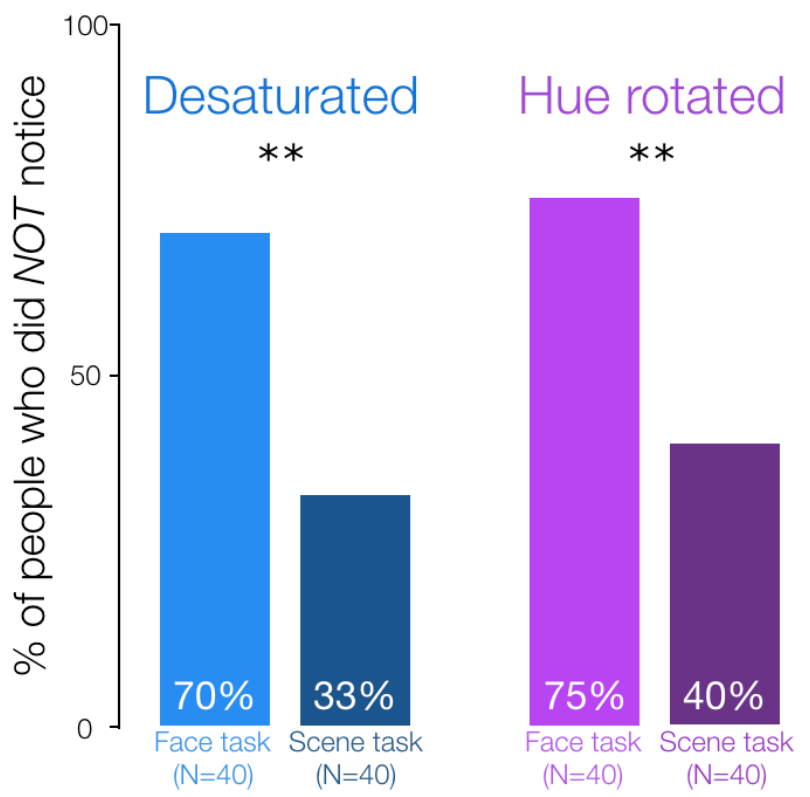

Figure 4. Inattentional blindness rates for each experimental condition. The percentage of participants who failed to notice the critical stimulus is plotted on the vertical axis. Each bar corresponds to a different experimental condition. ${ }^{* *} P<0.01$.

As mentioned above, the last step in our experimental procedure was showing participants four examples of different critical stimuli in order to 1) verify that they did not notice the stimulus alterations and 2) determine if they could identify the last image they saw even if they were unaware of the critical manipulations. How often do participants correctly identify the last image they saw? In this case, the percent of participants correctly recognized the critical stimulus was quite high for both tasks (face task: 85\%, 34/40 participants; scene task: $87.5 \%, 35 / 40$ ). What are the inattentional blindness rates when only examining the participants that correctly identified the last image from four alternatives? Here, we found that the inattentional blindness rates were virtually identical when only examining those participants (face task: 68\%, 23/34 participants; scene task: $29 \%, 10 / 35)$. For these results, the key point is that even though participants processed enough of the critical stimulus to pick it out amongst four options, they were unaware of the fact that the stimuli were desaturated in the periphery.

Meanwhile, with hue rotated critical stimuli, we found a similar pattern of results. During the face task, $75 \%$ of participants (30/40) were unaware of the color rotation in the periphery, while $40 \%$ of participants (16/40) were unaware of the color rotation in the periphery during the scene task. As was the case with desaturated critical stimuli, there was a significant effect of task $\left(X^{2}(1)=8.64, P<0.01\right)$. Finally, we once again asked what percentage of participants could identify the critical stimulus during the four alternative forced choice task and how the inattentional blindness rates varied accordingly. In this case, we found that the majority of participants could identify the critical stimulus (face task: $87.5 \%, 35 / 40$ participants; scene task: $87.5 \%, 35 / 40$ ) and the inattentional blindness rates were very similar when only examining those participants (face task: 74\%, 25/35 participants; scene task: 34\%, 12/35). 


\section{Discussion}

Here, we examined how much color information could be processed "in the blink of an eye." Overall, we found that when attention was centered near fixation with the face task, a majority of observers were entirely unaware of drastic alterations to the visual periphery. Moreover, even when attention was deployed more towards the periphery with the scene task, more than a third of observers failed to notice these peripheral alterations. Taken together, these results highlight how the immediate percept of a single fixation is shockingly impoverished and lacks a surprising amount of color. These findings raise a variety of natural questions, which we discuss below.

First, how do these results relate to the fact that there are fewer color-responsive cone cells in the periphery of the retina (Curcio et al., 1990)? Is this what drives our effects? Are people entirely colorblind in the periphery? No. It is often mistakenly claimed that humans cannot perceive color in the periphery. This is incorrect and humans do have some color perception abilities in the periphery (Hansen et al., 2009; Webster et al., 2010; Tyler, 2014). Although cones in the periphery are less dense than in the fovea, they are not entirely absent and can support some color perception (Rosenholtz, 2016). However, these behavioral effects usually require highly artificial situations where observers are directing all of their attention towards the periphery while looking at a simple monochromatic stimulus in isolation. Here, we tried to create experimental conditions that more closely mimicked real-world viewing. Specifically, we chose primary tasks that are somewhat similar to tasks performed in everyday life (i.e., object recognition at the fovea and broad judgments about a scene). The idea behind these tasks was to examine color perception when participants' attention is deployed in more ecologically valid conditions relative to when their attention is entirely in the periphery, as is the case in other studies on peripheral color vision. In addition, rather than use simple isolated color stimuli, we chose to use images of natural scenes as our stimuli since there are many factors that limit color perception in natural scene conditions in everyday life that are not present with isolated stimuli (e.g., color crowding; van den Berg et al., 2007; Kennedy \& Whitaker, 2010; Whitney \& Levi, 2011). Thus, even though observers do have the ability to recognize some color in the periphery, our results with more ecologically valid tasks and natural scenes suggest that a snapshot of perception in everyday life is surprisingly sparse.

One likely factor contributing to our results is the fact that we presented observers with brief snapshots of unrelated, individual images. In everyday life, however, observers are not presented with stimuli in this manner; instead, they are embedded within a scene for longer periods of time and, on average, sequentially fixate upon different locations several times a second (Rayner, 1998; Henderson, 2003). The integration of these successive fixations into a stable perceptual representation has been thoroughly studied and is referred to in the literature as transsaccadic integration (or transsaccadic memory; Irwin, 1996; Hollingworth et al., 2008; Melcher \& Colby, 2008; Cavanagh et al., 2010; Rolfs et al., 2015). It is transsaccadic integration that leads to the sense of perceptual continuity even though the visual world is constantly being sampled in discrete fixations. With this research in mind, it is natural to wonder about the role transsaccadic integration plays in forming the subjective impression of a perceptual experience that is more colorful than our data suggests. Under this view, although each independent sampling of the visual world lacks 
much color in the periphery, those samples are stitched together to create an experience of color across the visual world via color-percept integration. Going forward, future research could explore this possibility by directly investigating the role of transsacadic integration in color perception.

Another way in which multiple saccades within a scene may lead to our intuitive sense of color stems from the possibility that color is filled-in from memory and experience over time. As observers gaze upon a scene, they may store information from each successive fixation to form a detailed representation of the world around them. Then, when observers move their gaze to new parts of the scene, and those previously foveated objects are now in the periphery, the color of those items may be filled-in from these representations that have been built up over time. Indeed, examples such as the uniformity illusion (Otten et al., 2016), Troxler fading (Martinez-Conde et al., 2006), and color spreading (Nakayama et al., 1990; Bressan et al., 1997) all demonstrate how the visual system can fill-in certain aspects of the world. Could such mechanisms lead to the impression of color throughout the visual world in naturalistic settings? While this is certainly an intriguing possibility, we are somewhat skeptical of this idea. Although there are numerous examples of filling-in with a wide variety of stimuli, one thing those stimuli all have in common is that they are all extremely simple and artificial. Indeed, the neural mechanisms that have been linked with perceptual filling-in have all been consistently found within the earlier parts of visual processing (e.g., LGN, V1, V2, MT, etc. Komatsu, 2006). At this time, however, there is no behavioral or neural evidence of filling-in with complex real-world stimuli. In fact, it is difficult to imagine how such a process could work with natural scenes since the world is often full of numerous objects that are different colors (Balas \& Sinha, 2007). How would the visual system know which colors to assign to which objects when presented with a single image for a few hundred milliseconds? Thus, we believe that it is unlikely that a higher-level filling-in mechanism is what provides observers with the impression of a richly detailed color experience.

Finally, an important metacognitive question behind all of these ideas focuses on reconciling the findings reported here with personal introspection. Specifically, why do our results suggest we see so little color, when intuition suggests we see far more (Block, 2007; Haun et al., 2017; Tyler, 2015)? While we cannot offer a definitive answer to this question, since our data do not directly speak to this particular question, pursuing the different possibilities described above (i.e., transsacadic integration, perceptual filling-in, etc.) could lend insight into how the subjective impression of a richly colorful experience arises from very limited samples. Regardless of the specific mechanisms that work together to create the perceptual experience of color, it is important that researchers are careful to distinguish between "perceptual experience" and "perceptual beliefs." To make this distinction clear, consider a coffee mug on your desk that is off to the periphery. Now imagine that you first look at your computer monitor, then you look over to the mug, and then back to the monitor. If a moment later you are asked about the color of the mug, you will have no problem reporting its color (e.g., blue). But would you correctly answer that question because you were actively having an actual percept of the color blue, or because you simply remember that the mug is blue even if you are not experiencing blue in that moment? In one case, you actually see/experience the color of that item and can therefore accurately report its color. In the other case, you do not see/experience the item in that 
moment at all, but have prior knowledge/beliefs about the world that allow you to reach the correct answer. Going forward, it will be critical to differentiate between these different factors when trying to characterize the nature of perceptual experience. While they are intimately related, there are important differences between a person's experience of the world and their beliefs about the world and those differences must be kept in mind when trying to understand how observers form the metacognitive beliefs about their perceptual experiences.

Overall, we believe our results suggest that a single snapshot of perceptual experience lacks a surprising amount of color. Moreover, the fact that the inattentional blindness rates vary as a function of the task being performed (i.e., face vs. scene task) suggests that the richness of color perception is not a static constant. Instead, the extent of color perception likely varies from moment to moment as a function of attention. This idea is important for general debates about the richness of perception and the contents of perceptual awareness (Block, 2007; Cohen \& Dennett, 2011).

\section{Supplemental materials}

Data reported in Results section (but broken down for each individual participant and condition) are available on the Open Science Framework at https://osf.io/yb3dz/. Supplementary figures describing the stimulus generation procedures and more examples of our critical stimuli can be found online.

\section{Acknowledgements}

This study was supported by a National Science Foundation Collaborative Research Award (\#1829470) to M.A.C. Thanks to Rachel Albert, Thomas Botch, Sarah Cormiea, Leyla Isik, Nancy Kanwisher, Ken Nakayama, and Caroline Robertson for extensive discussion and feedback on the project. Thanks to Sebastian Montesinos and Caroline Ostrand for help with data collection.

\section{References}

Balas, B. \& Sinha, P. (2007). "Filling-in" colour in natural scenes. Visual Cognition. 15, 765-778.

Block, N. (2007). Consciousness, accessibility, and the mesh between psychology and neuroscience. Behavioral and Brain Sciences, 30, 481-499.

Brainard, D.H. (1997). The Psychophysics Toolbox. Spatial Vision, 10, 433-436.

Bressan, P., Mingolla, E., Spillman, L., \& Watanabe, T. (1997). Neon color spreading: A review. Perception, 26, 1353-1366.

Castelhano, M.S., \& Heaven, C. (2011). Scene context influences without scene gist: Eye movements guided by spatial associations in visual search. Psychonomic Bulletin and Review, 18, 890-896. 
Cavanagh, P., Hunt, A.R., Afraz, A., \& Rolfs, M. (2010) Visual stability based on remapping of attention pointers. Trends in Cognitive Sciences, 14, 147-153.

Cohen, M.A. \& Dennett, D.C. (2011). Consciousness cannot be separated from function. Trends in Cognitive Sciences, 15, 358-364.

Curcio, C.A., Sloan, K.R., Kalina, R.E., \& Hendrickson, A.E. (1990). Human photoreceptor topography. Journal of Comparative Neurology, 292, 497-523.

Haun, A.M., Tononi, G., Koch, C., \& Tsuchiya, N. (2017). Are we understanding the richness of visual experience? Neuroscience of Consciousness, 1, doi: https://doi.org/10.1093/nc/niw023

Hansen, T., Pracejus, L., \& Gegenfurtner, K.R. (2009) Color perception in the intermediate periphery of the visual field. Journal of Vision, 9(4), 26.

Hayhoe, M.M., Shrivastava, A., Mruczek, R., \& Pelz, J. (2003). Visual memory and motor planning in a natural task. Journal of Vision, 3, 49-63.

Henderson, J.M. (2003). Human gaze control during real-world scene perception. Trends in Cognitive Sciences, 7, 498-504.

Hollingworth, A., Richard, A.M., \& Luck, S.J. (2008) Understanding the function of visual short-term memory: Transsacadic memory, object correspondence, and gaze correction. Journal of Experimental Psychology: General. 137, 163-181.

Irwin, D.E. (1996) Integrating information across saccadic eye movements. Current Directions in Psychological Science, 5, 94-100.

Kennedy, G.J. \& Whitaker, D. (2010). The chromatic selectivity of visual crowding. Journal of Vision, 10, 1-13.

Komatsu, H. (2006). The neural mechanisms of perceptual filling-in. Nature Reviews Neuroscience, 7, 220-231.

Mack, A. \& Rock, I. (1998). Inattentional Blindness. MIT Press: Cambridge.

Martinez, Conde, S., Macknick, S.L., Troncoso, X.G., \& Dyar, T.A. (2006) Microsaccades counteract visual fading during fixation. Neuron, 49, 297-305.

Melcher, D., \& Colby, C.L. (2008) Transsaccadic perception. Trends in Cognitive Sciences, 12, 466-473.

Millodot, M. (2014) Dictionary of Optometry and Visual Science. Elsevier Health Sciences: United Kingdom. 
Nakayama, K., Shimojo, S., \& Ramachandran, V.S. (1990) Transparency: relation to depth, subjective contours, luminance, and neon color spreading. Perception, 19, 497-513.

Nuthmann, A. (2017). Fixation durations in scene viewing: Modeling the effects of local image features, oculomotor parameters, and task. Psychonomic Bulletin and Review, 24, 370-392.

Otten, M., Pinto, Y., Paffen, C.L.E., Seth, A.K., \& Kanai, R. (2016). The uniformity illusion: Central stimuli can determine peripheral perception. Psychological Science, 28, 56-68.

Pelli, D.G. (1997). The VideoToolbox software for visual psychophysics: transforming numbers into movies. Spatial Vision, 10, 437-442.

Pelz, J.G., \& Canosa, R. (2001). Oculomotor behavior and perceptual strategies in complex tasks. Vision Research, 41, 3587-3596.

Polyak, S.L. (1941) The Retina. University of Chicago Press: Chicago.

Rayner, K. (1998). Eye movements in reading and information processing: 20 years of research. Psychological Bulletin, 124, 372-422.

Rayner, K., Smith, T.J., Malcolm, G.L., \& Henderson, J.M. Eye movements and visual encoding during scene perception. (2008). Psychological Science, 20, 6-10.

Rensink, R., O'Regan, J.K., \& Clark, J.J. (1997). To see or not to see: The need for attention to perceive changes in scenes. Psychological Science, 8, 368-373

Rolfs, M. (2015). Attention in active vision: A perspective on perceptual continuity across saccades. Perception, 44, 900-919.

Rosenholtz, R. (2016). Capabilites and limitations of peripheral vision. Annual Reviews of Vision Science, 2, 437-457.

Swanson, W.H., \& Fish, G.E. (1995) Color matches in diseased eyes with good acuity: detection of deficits in cone optical density and in chromatic discrimination. Journal of the Optical Society of America, 12, 2230-2236.

Tyler, C.W. (2015) Peripheral color demo. iPerception, 6, 1-5.

Unema, P.J.A., Pannasch, S., Joos, M., \& Velichkovsky, B.M. (2005). Time course of information processing during scene perception: The relationship between saccade amplitude and fixation duration. Visual Cognition, 12, 473-494.

van den Berg, R., Roerdink, J.B.T.M., \& Cornelissen, F.W. (2007). On the generality of crowding: Visual crowding in size, saturation, and hue compared to orientation. Journal of Vision. 7, 1-11. 
Webster, M.A., Halen, K., Meyers, A.J., Winkler, P., \& Werner, J.S. (2010). Colour appearance and compensation in the near periphery. Proceedings of the Royal Society $B$, $277,1817-1825$.

Whitney, D. \& Levi, D.M. (2011). Visual crowding: A fundamental limit on conscious perception and object recognition. Trends in Cognitive Sciences, 15, 160-168.

Xiao, J., Hays, J., Ehinger, K., Oliva, A., \& Torralba, A. (2010) SUN Database: Large-scale scene recognition from Abbey to Zoo. IEEE Conference on Computer Vision and Pattern Recognition

${ }^{1}$ All stimuli are available for download at http://www.michaelacohen.net/demosstimuli.html 